\title{
O uso de drogas psicotrópicas e a prevenção no Brasil
}

\author{
Use and prevention of psychotropic drugs \\ in Brazil
}

Ana Regina Noto 1

José Carlos F. Galduróz 1

\footnotetext{
${ }^{1}$ Centro Brasileiro de Informações sobre Drogas Psicotrópicas, Departamento de Psicobiologia, Escola Paulista de Medicina, Universidade Federal de São Paulo, Rua Botucatu 862, 1o andar, 04023-062 São Paulo, SP, Brasil. cebrid@psicobio.epm.br
}

\begin{abstract}
Even though the context related to the use of psychotropic drugs in Brazil is still somewhat unknown, available studies point to alcohol, tobacco and some psychotropic medications as the most used drugs and as responsible for the highest indexes of problems in our population. However, the increasing consumption of cocaine over the years is unquestionable, as well as the increase of a series of associated problems, including violence. As far as possible interventions in this scenario are concerned, the need for complementary preventive measures has been stressed. Although the repressive approach has been highlighted in the last decades, studies have pointed out to the limitations of this kind of intervention. The different levels of psychotropic drugs use prevention are presented in this study, along with comments on the main approaches used and how they have been implemented in Brazil.
\end{abstract}

Key words Psychotropic; Prevention; Drug Abuse; Public Policies
Resumo Embora o contexto relacionado ao consumo de drogas psicotrópicas no Brasil ainda seja pouco conhecido, os estudos disponiveis apontam que o álcool, o tabaco e alguns medicamentos psicotrópicos são as drogas mais consumidas e responsáveis pelos maiores indices de problemas para a nossa população. No entanto, é inegável o crescente consumo de cocaína ao longo dos últimos anos, bem como o aumento de uma série de problemas daí decorrentes, incluindo a violência. No que diz respeito às possíveis intervenções nesse cenário, embora o enfoque repressivo tenha recebido destaque nas últimas décadas, estudos têm apontado as limitações desse tipo de intervenção, enfatizando a necessidade de medidas preventivas complementares. No presente artigo são apresentados os diferentes niveis de prevenção ao uso de drogas psicotrópicas, sendo comentados os principais enfoques utilizados e como os mesmos vêm sendo implementados no Brasil.

Palavras-chave Psicotrópicos; Prevenção; Abuso de Drogas; Políticas Públicas 


\section{O uso de drogas psicotrópicas no Brasil}

O Brasil, maior país da América Latina, tem uma população de aproximadamente $158 \mathrm{mi}$ lhões de habitantes e é a oitava economia mundial. Entretanto, a maioria da população não tem acesso às riquezas geradas no país. Cerca de $15 \%$ da população é composta por adultos analfabetos e grande parte das crianças não tem acesso às necessidades básicas. As diferenças regionais do país são marcantes, refletindo-se em peculiaridades culturais que envolvem desde o nível de educação às práticas religiosas (IBGE, 1997).

Essa variabilidade também se reflete no cenário do consumo de drogas psicotrópicas, chegando a ser marcante para algumas drogas específicas. O consumo de cocaína é um exemplo típico, concentrando-se em algumas regiões do país, em especial no Sudeste e no Sul do Brasil, sendo mais comum em algumas populações específicas e praticamente inexistente em outras (Nappo et al., 1996; Nappo, 1996a, Noto et al., 1998). Por outro lado, vale considerar que existem drogas cujo consumo se distribui de forma mais uniforme no território nacional, como é o caso do álcool e do tabaco (Almeida-Filho et al., 1992; Noto \& Carlini, 1995; Galduróz et al., 1997).

Também merecem destaque as diferenças de padrão de consumo entre homens e mulheres, sendo as drogas ilícitas (maconha e cocaína) mais consumidas por homens, e os medicamentos psicotrópicos (ansiolíticos, anfetaminas, entre outros) preferidos pelas mulheres (Nappo \& Carlini, 1993; Nappo, 1996b; Galduróz et al., 1997; Nappo et al., 1998).

O conhecimento dessas peculiaridades é essencial para subsidiar as políticas públicas. No entanto, ainda é pouco conhecida a real dimensão do uso de drogas no Brasil, bem como os problemas decorrentes desse uso, especialmente devido à carência de estudos nessa área, o que se acentua diante das dificuldades relacionadas à clandestinidade que envolve o uso de drogas ilícitas.

Entre os poucos estudos epidemiológicos até hoje realizados no Brasil, destacam-se os levantamentos realizados pelo Cebrid (Centro Brasileiro de Informações sobre drogas Psicotrópicas) entre estudantes de dez capitais brasileiras, nos anos de 1987, 1989, 1993 e 1997, os quais indicam o álcool e o tabaco, como os psicotrópicos mais consumidos. Entretanto, excetuando estes, $24,7 \%$ dos estudantes entrevistados em 1997 relataram já ter ao menos experimentado drogas. Os inalantes (solventes) foram os psicotrópicos mais citados $(13,8 \%)$, seguidos pela maconha $(7,6 \%)$, pelos medicamentos ansiolíticos $(5,8 \%)$, anfetamínicos $(4,4 \%)$ e pela cocaína (2\%) (Galduróz et al., 1997).

Paralelamente, estudos realizados nos anos de 1987, 1989, 1993 e 1997 entre crianças e adolescentes em situação de rua de seis capitais brasileiras têm indicado um consumo de psicotrópicos muito superior aos valores observados entre estudantes. No ano de 1997, $88,1 \%$ dos entrevistados relataram já ter usado drogas pelo menos uma vez na vida, sendo os inalantes e a maconha as drogas mais citadas (excetuando o álcool e o tabaco). No que diz respeito às demais drogas, foram observadas diferenças regionais, aparecendo em destaque a cocaína nas capitais do Sul e do Sudeste, e os medicamentos psicotrópicos nas capitais do Nordeste. Esses estudos também têm apontado uma estreita relação entre o consumo de psicotrópicos e outras atividades ilícitas como a prática de furtos (Forster et al., 1992, 1996; Noto et al., 1997, 1998).

No que diz respeito aos atendimentos hospitalares provocados pelo abuso de psicotrópicos, levantamentos realizados junto a hospitais e clínicas psiquiátricas apontam o álcool como responsável por cerca de $90 \%$ das internações por dependência. Esses estudos também mostram que as internações por cocaína vêm aumentando gradativamente desde 1987, bem como as por maconha vêm diminuindo. Essas mudanças alteraram o perfil das internação e, atualmente, a cocaína ocupa o primeiro lugar entre as drogas ilícitas, posto este ocupado pela maconha até 1991 (Noto \& Carlini, 1995).

As drogas psicotrópicas também assumem um papel de destaque no cenário dos acidentes de trânsito. Um estudo da Associação Brasileira dos Departamentos de Trânsito (Abdetran), envolvendo quatro capitais brasileiras: Salvador, Recife, Brasília e Curitiba, detectou que em $27,2 \%$ dos casos analisados de vítimas de acidentes de trânsito, a dosagem de álcool no sangue excedia o valor de $0,6 \mathrm{~g} / \mathrm{l}$, limite permitido pelo novo Código Nacional de Trânsito. Entre os demais psicotrópicos, destacaramse a maconha (detectada em 7,7\% dos casos), os benzodiazepínicos $(3,4 \%)$ e a cocaína $(2,3 \%)$ (Abdetran, 1997). 
Em relação ao tráfico de drogas, ao serem analisadas as apreensões realizadas pela Polícia Federal ao longo dos últimos anos, verifica-se que, enquanto as apreensões de maconha diminuíram a partir do final da década de 80 , as de cocaína aumentaram de maneira considerável. Embora as quantidades de drogas apreendidas tenham sido grandes, o número de inquéritos e indiciamentos foi pequeno quando comparado com outros países (Galduróz et al., 1994; Noto et al., 1995).

Segundo o Drug Enforcement Administration, o Brasil é atualmente a principal rota de tráfico de cocaína na América Latina, situação que acarreta problemas sociais consideráveis para o nosso país, como a disseminação da Aids e a violência.

Nesse contexto, torna-se evidente a crescente participação da cocaína no cenário brasileiro, situação que merece atenção especial das políticas públicas na área (Carlini et al., 1995; Dunn et al., 1996; Fonseca \& Issy, 1996; Nappo et al., 1996).

É importante assinalar que o álcool, o tabaco e alguns medicamentos psicotrópicos (especialmente ansiolíticos e anfetaminas), embora não tão alardeados, continuam sendo as drogas mais consumidas e as que trazem os maiores prejuízos à população brasileira. No entanto, ainda são muito pouco consistentes as intervenções preventivas voltadas para essas drogas, deixando aberto espaço para campanhas publicitárias cada vez mais sofisticadas para a promoção do consumo que mascaram os inúmeros problemas sociais que envolvem o abuso do álcool e do tabaco.

Num país como o Brasil, onde a maioria da população tem a mídia como principal fonte de informações, o que é divulgado pelos meios de comunicação de massa passa a ser padrão de verdade. Assim, em contrapartida à negligência política no que diz respeito às drogas lícitas, para estas imperam os alardes da mídia, que vêm criando um "pânico", inclusive dificultando algumas intervenções específicas (Carlini-Cotrim et al., 1995).

Ainda vale ressaltar que a violência relacionada ao uso abusivo de drogas não fica restrita aos acidentes de trânsito, às cenas de brigas em bares ou entre traficantes, mas também envolve o ambiente familiar de forma considerável. Ainda são raros os estudos sobre essa questão no Brasil.
A estrutura governamental e a política nacional na área

As incoerências citadas anteriormente são reflexos das inúmeras divergências que envolvem a questão dos psicotrópicos e, especialmente, da falta de uma política pública integrada. Os órgãos governamentais, na maioria das vezes, atuam isoladamente e dificilmente conseguem traduzir suas propostas em ações práticas. Dessa maneira, embora sejam relativamente freqüentes os discursos políticos, as palestras, os simpósios e, até mesmo os encontros científicos sobre o tema, são poucas as intervenções preventivas implementadas de fato. Nesse contexto, as poucas propostas governamentais que conseguiram atingir um estágio mais avançado de implementação ficaram fragilizadas no processo de mudança de governo, ou até mesmo acabaram sendo totalmente substituídas sem qualquer fundamento científico, como, por exemplo, o Projeto Valorização da Vida (Rio Grande do Sul) e o Projeto Escola é Vida (São Paulo). No entanto, parece estar em curso um processo de mudança nesse contexto, uma vez que o número e a qualidade das intervenções vêm aumentando, ainda que discretamente, ao longo dos últimos anos.

Considerando a estrutura política no nível nacional, o Conselho Federal de Entorpecentes (Confen), ligado ao Ministério da Justiça, foi o órgão responsável pela coordenação das políticas públicas na área de drogas psicotrópicas no período de 1980 a 1998. Recentemente, foi criada a Secretaria Anti-drogas (Senad), subordinada diretamente à Presidência da República, que está dando os primeiros passos para estabelecer uma nova política pública no campo das drogas, tendo como desafio integrar as diferentes ações nessa área.

No que diz respeito especificamente à área da educação, o Ministério da Educação tem a responsabilidade de estabelecer campanhas e atividades de prevenção ao uso de drogas psicotrópicas dentro de um sistema formal de educação. No entanto, embora seja crescente o interesse nessa área, na prática ainda pouco se tem avançado.

$\mathrm{Na}$ área de saúde, o Ministério conta com a Coordenação Nacional de Saúde Mental (Cosam) e ainda com a Coordenação Nacional de Doenças Sexualmente Transmissíveis (DST) e Aids. Ambas coordenam projetos voltados para prevenção, tratamento e, mais recentemen- 
te, redução de danos relacionados ao uso de drogas psicotrópicas.

Confrontando a política nacional e o cenário epidemiológico no Brasil ao longo dos últimos anos, é possível concluir que embora mudanças políticas tenham ocorrido, o quadro epidemiológico não sofreu grandes alterações. As poucas que aconteceram, em geral, foram para pior, especialmente no que se refere ao aumento do consumo e problemas relacionados às drogas ilícitas. Esse contexto sugere que as medidas adotadas nestes últimos anos não parecem ter tido a eficácia esperada e, portanto, torna-se essencial estudar formas alternativas de lidar com a questão.

\section{Os diferentes níveis de prevenção}

Tratando-se de psicotrópicos, as intervenções repressivas e de controle foram as que receberam maior destaque ao longo das últimas décadas. Esse tipo de vertente teve seu auge na década de 80, no movimento norte-americano denominado Guerra às Drogas, que se caracterizou por um enfoque alarmista, intolerante e repressivo. Esse movimento também teve como meta a "exportação" dessa postura para países menos desenvolvidos, principalmente aqueles considerados como rotas de tráfico, dentre os quais o Brasil (Carlini-Cotrim, 1995).

Diversos estudos têm apontado as inúmeras limitações e complicações relacionadas às medidas proibitivas como a restrição à venda de medicamentos psicotrópicos, a repressão ao tráfico de drogas ilícitas, o controle policial voltado aos usuários e outros. A restrição ao acesso a determinada droga, enquanto medida isolada, pode diminuir o consumo da mesma, porém, em geral, desencadeia um processo praticamente imediato de substituição daquela substância por outras mais disponíveis (Westermeyer, 1976; Schottstaedt \& Bjork, 1977; Carlini-Cotrim \& Silva-Filho, 1988; Noto et al., 1998).

Dessa maneira, torna-se essencial o desenvolvimento de intervenções complementares, voltadas para a prevenção, incluindo trabalhos direcionados para os demais aspectos que envolvem o uso de drogas, ou seja, o indivíduo e o meio social em que se encontra.

As ações preventivas ao uso indevido de drogas podem acontecer em diferentes níveis, dependendo da população-alvo e do perfil da intervenção.
Prevenção primária - É o conjunto de ações que procuram evitar a ocorrência de novos casos de uso abusivo de psicotrópicos ou até mesmo um primeiro uso (OMS, 1992).

Esse tipo de intervenção pode ser realizada dentro de diferentes enfoques, sendo a divulgação de informações o mais conhecido. Dentro dos modelos informativos, destacam-se duas vertentes principais: o modelo baseado no amedrontamento (divulgação apenas dos prejuízos causados pelas drogas), que é atualmente considerado inadequado, embora já tenha sido muito utilizado em passado recente; e o modelo baseado na informação científica não tendenciosa (informação geral e isenta sobre as drogas), que vem sendo muito utilizado ao longo dos últimos anos. No entanto, estudos têm sugerido que, apesar da informação ser fundamental, quando aplicada isoladamente não tem tido muito sucesso enquanto medida preventiva, uma vez que embora ela seja capaz de mudar alguns conceitos, isso não implica, necessariamente, em uma mudança de comportamento (Carlini-Cotrim, 1992; Dorn \& Murji, 1992; Noto et al., 1998).

No entanto, existem outros modelos mais recentes de prevenção primária, cuja efetividade ainda não pode ser avaliada adequadamente. Nesse conjunto se incluem modelos que buscam fortalecer atitudes saudáveis e/ou a oferta de alternativas esportivas/culturais; modelos voltados para a modificação do ambiente, das condições e práticas instrucionais; e ainda alguns que visam a sensibilização de lideranças naturais para atuarem como multiplicadores do processo (Carlini-Cotrim, 1992; Dorn \& Murji, 1992). Este modelo de formação/sensibilização de multiplicadores vem sendo utilizado recentemente em algumas escolas brasileiras da rede pública em um projeto coordenado pela Coordenação Nacional de DST e Aids (Projeto Escolas), envolvendo os temas drogas, sexualidade, Aids e outras DST.

Diante da existência de inúmeros modelos de prevenção primária, vale salientar que o critério mais importante para escolher o mais adequado a cada circunstância e contexto é conhecer e respeitar as características e as necessidades da comunidade onde se pretende atuar.

No entanto, as campanhas até então realizadas no Brasil têm negligenciado este aspecto, iniciando projetos de prevenção primária sem qualquer estudo prévio da população em questão. Dessa forma, torna-se essencial incentivar esse tipo de estudo, bem como os de 
avaliação da efetividade das propostas implementadas.

Prevenção secundária - É o conjunto de ações que procuram evitar a ocorrência de complicações para as pessoas que fazem uso ocasional de drogas e que apresentam um nível relativamente baixo de problemas (OMS, 1992).

Essas ações buscam sensibilizar as pessoas a respeito dos riscos, favorecendo a mudança de comportamento através do aprendizado de novas atitudes e escolhas mais responsáveis (OMS, 1992; Mesquita et al.,1993).

Os serviços específicos de prevenção secundária ainda são muito pouco explorados no Brasil, especialmente em função das inúmeras dificuldades relacionadas à implementação desse tipo de intervenção. Os usuários não dependentes muitas vezes não notam qualquer prejuízo imediato do uso da droga e ainda sentem imenso prazer em usá-las. Dessa forma, eles não tendem a buscar ajuda em serviços especializados, e, dificilmente, são identificados. No caso das drogas ilícitas, o reconhecimento do usuário torna-se ainda mais complicado por tratar-se de um comportamento clandestino.

Entre as poucas iniciativas de intervenção secundária em nosso país, destaca-se o projeto pioneiro desenvolvido pela Unidade de Dependência de Drogas da Unifesp, tendo como referencial uma técnica de base cognitiva desenvolvida no Canadá (Formigoni, 1992).

Prevenção terciária - É o conjunto de ações que, a partir de um problema existente, procura evitar prejuízos adicionais e/ou reintegrar na sociedade os indivíduos com problemas sérios. Também busca melhorar a qualidade de vida dos usuários junto à família, ao trabalho e à comunidade de uma forma geral (OMS, 1992).

Na prática, essas ações envolvem o identificar e o lidar com casos emergenciais (como síndrome de abstinência, overdose, tentativas de suicídio, etc.) e/ou com pacientes portadores de problemas que necessitam encaminhamento (hepatite, Aids, cirrose, entre outros). Também envolvem a orientação familiar e o auxílio na reabilitação social dos usuários.

As ações de prevenção terciária muitas vezes se mesclam com as ações inerentes ao tratamento daqueles usuários que buscam ajuda para sua recuperação. Existem inúmeros modelos de tratamento para dependência química, incluindo várias linhas de grupos de autoajuda (com destaque para os Alcoólicos Anônimos), abordagens psicanalíticas, comporta- mentais, cognitivas, medicamentosas. No entanto, os diversos estudos comparativos dessas abordagens não têm indicado diferença de efetividade entre as mesmas. Ao contrário, os índices são muito semelhantes e baixos. A maioria dos estudos refere um baixo índice de abstinentes após o tratamento, sendo poucos os programas que atingem $35 \%$ de recuperação após dois anos de acompanhamento dos pacientes (Smart, 1970; McLellan et al., 1983; Formigoni \& Neumann, 1992; Miller, 1992).

Especialistas nessa área têm buscado formas alternativas para aumentar esses índices de recuperação. Alguns defendem a importância de um pareamento entre os pacientes e as abordagens terapêuticas, ou seja, que a escolha do tratamento tenha como referência as características/necessidades do paciente. Essa linha de estudos tem sido um dos principais focos de pesquisas sobre o tratamento da dependência química ao longo dos últimos anos (Lindstrom, 1992).

No entanto, é consenso a crença na necessidade da criação de redes de assistência integral ao dependente; incluindo serviços de orientação familiar, encaminhamento para tratamento de comorbidades, apoio para reinserção profissional e/ou educacional.

Redução de danos secundários ao uso indevido de drogas - Essa categoria diz respeito a políticas públicas que buscam reduzir os efeitos negativos decorrentes do uso de drogas, tanto para o indivíduo quanto para a sociedade.

Dessa forma, as políticas de redução de danos reconhecem que as pessoas usam e muitas delas continuarão usando drogas, independentemente das intervenções convencionais, que em geral apresentam baixa efetividade.

Essa estratégia de saúde pública vem recebendo maior atenção a partir da constatação da participação do consumo de drogas na transmissão do HIV e das hepatites virais pela troca de agulhas e seringas contaminadas durante a prática de uso injetável de drogas (O’Hare, 1994; Rhodes et al., 1996).

Embora esse uso já tivesse sua importância reconhecida internacionalmente, no Brasil foram inúmeras as resistências políticas para a implementação de projetos voltados para a redução de danos.

Entre as iniciativas pioneiras no Brasil, destacam-se os estudos e projetos desenvolvidos em Santos, Rio de Janeiro e Salvador (Lima et al., 1994; Mesquita, 1994; Telles, 1994; Andra- 
de \& Lemos, 1998). Essas iniciativas ampliaram a participação do Brasil dentro do cenário internacional de redução de danos, culminando na realização da 9th International Conference on the Reduction of Drug Related Harm, no ano de 1998 em São Paulo.

\section{Considerações finais}

Conforme mencionado no presente artigo, no Brasil as intervenções preventivas não têm tradição no que diz respeito ao uso abusivo de psicotrópicos. Ao contrário, as iniciativas, em geral, são isoladas; as pesquisas são pouco valorizadas; e os primeiros estudos brasileiros de avaliação de efetividade das intervenções surgiram apenas nas duas últimas décadas e ainda são muito pouco freqüentes. Porém, parece existir uma tendência à mudança desse panorama, uma vez que o interesse e o número de projetos de prevenção nessa área vem aumentando. A estruturação e, especialmente, a integração dos diferentes níveis de intervenção constituem-se o grande desafio para os próximos anos.

\section{Referências}

Almeida-Filho N, Mari JJ, Coutinho E, França JF, Fernandes JG, Andreoli SB, Busnello ED 1992. Estudo multicêntrico de morbidade psiquiátrica em áreas urbanas brasileiras (Brasília, São Paulo, Porto Alegre). Revista ABP-APAL 14(3): 93-104.

Andrade TM \& Lemos SRM 1998. Textos Orientados para a Assistência à Saúde entre Usuários de Drogas. Centro de Estudos e Terapia do Abuso de Drogas, Universidade Federal da Bahia, Salvador.

Associação Brasileira dos Departamentos de Trânsito 1997. Impacto do Uso do Álcool e Outras Vítimas de Acidentes de Trânsito. Cetad \& Raid, 87 pp.

Carlini-Cotrim B 1992. A Escola e as Drogas: Realidade Brasileira e Contexto Internacional. Tese de Doutorado, Pontifícia Universidade Católica de São Paulo, São Paulo.

Carlini-Cotrim B 1995. Movimentos e discursos contra as drogas: o caso da sociedade norte-americana. $R e-$ vista ABP-APAL 17(3): 93-101.

Carlini-Cotrim B \& Silva-Filho AR 1988. O abuso do Artane" por meninos de rua de São Paulo. Possíveis influências da Portaria no 27/86 da DIMED. Jornal Brasileiro de Psiquiatria 37 (4): 201-203.

Carlini-Cotrim B, Galduróz JCF, Noto AR, Pinsky I 1995. A mídia na fabricação de pânico de drogas: um estudo no Brasil. Comunicação e Política 1(2): 217 230.

Carlini EA, Galduróz JCF, Noto AR, Nappo SA, Lima E, Adiala JC 1995. Revisão: perfil de uso da cocaína no Brasil. Jornal Brasileiro de Psiquiatria 44(6): 287 303.
Dorn N \& Murji K 1992. Drug Prevention: a Review of the English Language Literature. Research Monograph 5, Institute for the Study of Drug Dependence, $46 \mathrm{pp}$.

Dunn J, Laranjeira RR, Silveira DX, Formigoni MLOS, Ferri CP 1996. Crack cocaine: an increase in use among patients attending clinics in São Paulo: 19901993. Substance Use \& Misuse 31(4): 519-527.

Formigoni MLOS 1992. Histórico e apresentação da estrutura do projeto, p. 15-23. In MLOS Formigoni, Intervenção Breve na Dependência de Drogas: a Experiência Brasileira. Contexto, São Paulo.

Formigoni MLOS \& Neumann BRG 1992. Comparação da efetividade do tratamento de dependentes de álcool e outras drogas pela técnica de intervenção breve e psicoterapia de grupo, p. 119-141. In MLOS Formigoni, Intervenção Breve na Dependência de Drogas: a Experiência Brasileira. Contexto, São Paulo.

Forster LMK, Tannhauser M, Barros HMT 1996. Drug use among street children in southern Brazil. Drug and Alcohol Dependence 43(1,2): 57-62.

Fonseca MA \& Issy J-Merla 1996. In CRM Oliveira \& MA Fonseca, Consumo de Drogas no Distrito Federal. Conen-DF, Brasília, 75 pp.

Forster LMK, Barros HMT, Tannhauser SL, Tannhauser M 1992. Meninos na rua: relação entre abuso de drogas e atividades ilícitas. Revista ABP-APAL 14(3): 115-120.

Galduróz JCF, Figlie NB, Carlini EA 1994. Repressão às drogas no Brasil: a ponta do "iceberg"? Jornal Brasileiro de Psiquiatria 43(7): 367-371. 
Galduróz JCF, Noto AR, Carlini EA 1997. IV Levantamento sobre o Uso de Drogas entre Estudantes de 1 o e 2o Graus de 10 Capitais Brasileiras - 1997. Centro Brasileiro de Informações Sobre Drogas Psicotrópicas, Departamento de Psicobiologia, Universidade Federal de São Paulo, Escola Paulista de Medicina, $130 \mathrm{pp}$.

IBGE - Instituto Brasileiro de Geografia e Estatística 1997. Contagem da População 1996, v.1, Rio de Janeiro.

Lima ES, Friedman SR, Bastos FI, Telles PR, Friedmann P, Ward TP, Jarlais DC 1994. Risk factors for HIV-1 soroprevalence among injectors in the cocaine-using environment os Rio de Janeiro. Addiction 89: 689-698.

Lindstrom L 1992. Managing Alcoholism: Matching Clients to Treatment. Oxford University Press, New York, $373 \mathrm{pp}$.

MacLellan AT, Luborsky L, Woody GE, O’Brien CP, Druley KA 1983. Predicting response to alcohol and drug abuse treatments. Archives of General Psychiatry 40: 620-625.

Mesquita AMC, Halpern M, Buccaretchi HA 1993. Prevenção ao abuso de álcool e outras drogas. In AG Andrade, S Nicastre, E Tongue, Drogas: Atualização em Prevenção e Tratamento. Grea, Grupo Interdisciplinar de Estudos do Alcoolismo e Farmacodependência, São Paulo,

Mesquita F 1994. Perspectivas das estratégias de redução de danos no Brasil, p.169-180. In F Mesquita \& FI Bastos, Drogas e Aids: Estratégias de Redução de Danos. Hucitec, São Paulo.

Miller WR 1992. The effectiveness of treatment for substance abuse: reasons for optimism. Journal of Substance Abuse Treatment 9: 93-102.

Nappo SA 1996a. "Baquêros" e "Crackêros": um Estudo Etnográfico sobre o Consumo de Cocaína na Cidade de São Paulo. Tese de Doutorado, Universidade Federal de São Paulo, São Paulo.

Nappo SA 1996b. Consumption of anoretic amphetamine-like drugs (diethilpropion, fenproporex and mazindol) and of d,l-fenfluramine in Brazil during the years 1988 and 1989. Pharmacoepidemiology and Drug Safety 5: 19-25.

Nappo SA \& Carlini EA 1993. Preliminary finding: consumption of benzodiazepines in Brazil during the years 1988 and 1989. Drug and Alcohol Dependence 33: 11-17.
Nappo SA, Galduróz JCF, Noto AR 1996. Crack use in São Paulo. Substance Use \& Misuse 31(5): 565-759.

Nappo SA, Oliveira EM, Morosini S 1998. Inappropriate prescribing of compounded antiobesity formulas in Brazil. Pharmacoepidemiology and Drug Safety 7: 207-212.

Noto AR \& Carlini EA 1995. Internações hospitalares provocadas por drogas: uma análise de sete anos consecutivos (1987-1993). Revista ABP-APAL 17(3): 107-114.

Noto AR, Galduróz JCF, Carlini EA 1995. Maconha: uma avaliação da situação brasileira através de quatro parâmetros epidemiológicos. Revista ABP-APAL 17(4): 133-137.

Noto AR, Nappo SA, Galduróz JCF, Mattei R, Carlini EA 1997. Use of drugs among street children in Brazil. Journal of Psychoactive Drugs 29(2):185-192.

Noto AR, Nappo SA, Galduróz JCF, Mattei R, Carlini EA 1998. IV Levantamento sobre o Uso de Drogas entre Crianças e Adolescentes em Situação de Rua de Seis Capitais Brasileiras - 1997. Centro Brasileiro de Informações Sobre Drogas Psicotrópicas, Universidade Federal de São Paulo, Escola Paulista de Medicina, $120 \mathrm{pp}$.

O’Hare P 1994. Redução de danos: alguns princípios e ação prática, p. 65-78. In F Mesquita \& FI Bastos, Drogas e Aids: Estratégias de Redução de Danos. Hucitec, São Paulo.

OMS - Organização Mundial da Saúde 1992. Reagindo aos Problemas das Drogas e do Álcool na Comunidade, São Paulo.

Rhodes T, Hunter GM, Stimson GV, Donoghoe MC, Noble A, Parry J, Chalmers C 1996. Prevalence of markers for hepatitis B virus and HIV-1 among drug injectors in London: injecting careers, positivity and risk behaviour. Addiction 91(10): 1457-1467.

Schottstaedt MF \& Bjork JW 1977. Inhalant abuse in an indian boarding school. American Journal of Psychiatry 133:1290-1293.

Smart RG 1970. The evaluation of alcoholism-treatment programs. Addictions 17: 41-51.

Telles PR 1994. Estratégias de "redução de danos" e algumas experiências de Santos e Rio de Janeiro com usuários de drogas injetáveis, p. 181-190. In F Mesquita \& FI Bastos, Drogas e Aids: Estratégias de Redução de Danos. Hucitec, São Paulo.

Westermeyer J 1976. The pro-heroin effects of anti-opium laws in Asia. Archives of General Psychiatry 33: 1135-1139. 